\title{
Percutaneous Endoscopic Lumbar Discectomy versus Conventional Open Discectomy: A Prospective Randomized Comparative Trial
}

\author{
Ahmad Abdalla, Hazem Othman \\ Department of Neurosurgery, Assiut University, Assuit, Egypt \\ Email: pcahmadabdalla@yahoo.com
}

How to cite this paper: Abdalla, A. and Othman, H. (2019) Percutaneous Endoscopic Lumbar Discectomy versus Conventional Open Discectomy: A Prospective Randomized Comparative Trial. Open Journal of Modern Neurosurgery, 9, 105-114. https://doi.org/10.4236/ojmn.2019.92010

Received: January 6, 2019

Accepted: March 15, 2019

Published: March 18, 2019

Copyright $\odot 2019$ by author(s) and Scientific Research Publishing Inc. This work is licensed under the Creative Commons Attribution International License (CC BY 4.0).

http://creativecommons.org/licenses/by/4.0/

\begin{abstract}
Introduction: The majority of series considered the conventional open discectomy as the gold standard for the treatment of lumbar disc prolapse. Despite of the popularity of the lumbar endoscopic discectomy nowadays, many neurosurgeons still prefer conventional open discectomy. Purpose: Our study has been designed to compare between percutaneous endoscopic lumbar discectomy and conventional open discectomy; regarding surgical results, complications, clinical and functional outcomes. Patients and Methods: This study is a clinical prospective randomized controlled trial conducted upon 30 patients suffering from prolapsed lumbar disc, from December 2016 to May 2018. Those patients were divided randomly into 2 groups, 15 patients each. One group treated by percutaneous endoscopic interlaminar lumbar discectomy $(P E L D)$ and the other group treated by conventional open discectomy $(C O D)$. Operative time, wound size, Intraoperative blood loss, Intraoperative complications, postoperative hospital stay, Postoperative complication and the results of visual analogue score (VAS) and modified MacNab's criteria were assessed. Results: This study included 30 patients (18 males and 12 females). The mean age was about 35 years. Although, there was postoperative improvement of the VAS and MacNab's criteria in the two groups, there was no statistically significant difference between the preoperative and postoperative VAS of low back pain and radicular pain for the two groups in the follow up period. Conclusion: Both techniques give good results for patients; each technique has some advantages over the other.
\end{abstract}

\section{Keywords}

Lumbar Disc Prolapse, Open Discectomy, Endoscopic Discectomy 


\section{Introduction}

Lumbar disc prolapse has been the most common disabling spinal disease recorded in medical history [1]. The majority of series were considered the conventional open discectomy as the gold standard for the treatment of lumbar disc prolapse.

In 1977, Yasargil and Caspar introduced microdiscectomy which is now considered the gold standard [2].

In 1997, the endoscopic microdiscectomy was introduced; it allowed spinal surgeons to decompress a symptomatic lumbar nerve root by using an endoscopic minimally invasive surgical approach. This system offers many advantages over other minimally invasive surgical lumbar discectomy techniques: it reduces tissue trauma, allows direct visualization of the nerve root and disc diseases, and enables bony decompression.

Although the popularity of the lumbar endoscopic discectomy, may neurosurgeons still prefer conventional open discectomy [3].

Many reports have proved the efficacy of endoscopic microdiscectomy with overall comparable results [3]. But long term follow up is recommended to show whether this technique will improve upon the results of the gold standard procedures.

\section{Aim of the Work}

Our study has been designed to compare between percutaneous endoscopic lumbar discectomy and conventional open discectomy; regarding surgical results, complications, clinical and functional outcomes.

\section{Patients and Methods}

\subsection{Study Design}

This study is a clinical prospective randomized controlled trial conducted upon 30 patients suffering from prolapsed lumbar disc, from December 2016 to May 2018. Those patients were divided randomly into 2 groups, 15 patients each. One group treated by percutaneous endoscopic interlaminar lumbar discectomy $(P E L D)$ utilizing micro endoscopic tubular retractor system (EasyGo) and the other group treated by conventional open discectomy (COD), in Assiut University hospitals at Neurosurgery department.

\subsection{Inclusion Criteria}

The inclusion criteria were Postero-lateral, single level disc herniation at L4-5 or L5-S1 level with low back pain and unilateral radiculopathy and failure of conservative treatment for 12 weeks. We excluded patients with extraforaminal disc herniation, recurrent and/or multiple level discs prolapses and spondylolisthesis.

Preoperatively, all the patients were subjected to full history, general examination, neurological examination and Laboratory investigations; including CBC, 
bleeding profile, and blood chemistry. Neuroimaging investigations; including Plain x-ray radiography of lumbosacral spine and MRI films to evaluate disc level of herniation, disc height, disc degree of herniation, disc direction of herniation, and disc contour. Informed consents were signed by the patients themselves.

\subsection{Surgical Techniques}

All patients of both groups had general anesthesia. Knee chest prone position; the abdomen was watched free with cotton pads on pressure points. The back of the patient was shaved, cleansed with an Alcohol swab, sterilized with betadine and draped by sterile towels.

\subsubsection{Conventional Open Lumbar Discectomy}

Skin incision is performed midline. Dissection of muscle is best accomplished with a periosteal elevator and a fine electro cautery needle, exposing the wanted hemilamina and a portion of hemilaminae above or below to permit retraction without undue tension. A small bite in lamina, medially just at the base of spinous process, is done using kerrison, dissector is gently fixed in its site by hand. Lateral fluoroscopic imaging is done to confirm that we are on the targeted disc space.

Removal of bone starts at lower laminal margin overlying the involved disc space, performed with a small Kerriosn. A small fenestration is done in superior lamina till the superior border of ligamentum flavum starts to appear. After safe dissection from dura by dissector, the ligamentum flavum is opened by a dissector and scalpel, and then removal with a Kerrison. The nerve root is exposed well above and below the disc space. The nerve root is medially displaced at the disc space and the annulus is incised to remove the disc material.

Afterwards, the root and Dural sac are finally explored to check for any residual compression and/or retained sub-ligamentous disc fragments, controlling epidural bleeding with gel foam. Closure in layers was performed.

\subsubsection{Percutaneous Endoscopic Lumbar Discectomy}

The surgeon stands on the ipsilateral side of the herniated disc. The monitor of endoscope stands on the contralateral side opposite the upper half of the patient body. Anesthesia station is near the head of the patient. The flexible arm assembly is attached to the operating table rail ipsilateral to the disc herniation; the flexible arm holds the tubular retractor. The endoscope is then connected to the camera and to the light source, the white balance is done on the camera and then the orientation of directions and the degree of magnification is done.

After the operative field has been prepared and draped, the lumbar midline is palpated and marked with a surgical pen. A second line is drawn parallel to the first, approximately one fingerbreadth $(1.5 \mathrm{~cm})$ lateral to the midline on the side of the disc herniation. A steinmann pin was inserted into the paraspinal musculature targeting the disc level. The placement is confirmed using lateral fluoros- 
copy.

Then smallest soft tissue dilator is inserted over Steinmann pin, directed toward the inferior edge of the superior lamina then the pin removed. The next series of dilators are sequentially placed over each other. The optimum tubular retractor is placed over the sequential dilators and seated firmly on the bony anatomy. The flexible arm is attached to the bed rail and the selected tubular retractor. The flexible arm is secured and the sequential dilators are removed, establishing a tubular operative corridor.

The few muscle fibers that may be obscuring the way are coagulated by bipolar and removed. Bone removal begins on the infer-lateral portion of the superior lamina and may proceed to the superolateral portion of the inferior lamina using an electric drill and Kerrison punch. A fenestration is done in superior lamina till the superior border of ligamentum flavum starts to appear. Bone removal can be extended into the medial facet joint (partial medial facetectomy) to expose the nerve root, only when needed. The ligamentum flavum was opened and foraminotomy was done until we saw the proper root.

The axilla of the root is explored, root can be retracted medially. Annulotomy was done and free fragments or contained disc herniations are removed. Afterwards, the root and dural sac are finally explored to check for any residual compression and/or retained disc fragment, especially in sub-ligamentous area. Epidural bleeding is controlled with gel foam. The fascia is then closed by 2 simple interrupted sutures, then 2 subcutaneous inverted sutures are applied, the skin is closed with subcuticular sutures.

Postoperatively, all patients received intravenous antibiotic and analgesic. $\mathrm{Pa}-$ tients are discharged as soon as possible; as long as there are no complications.

\subsection{Outcome Measures}

All patients will be followed up at outpatient clinical after two weeks, one month, three months, six months, and yearly. The patients were evaluated clinically using Visual Analogue Score (VAS) for both low back pain and radicular pain and modified MacNab's criteria for patient satisfaction. Radiographic evaluation by plain $\mathrm{x}$-ray, CT and MRI lumbosacral spine were requested when indicated.

The comparison of the 2 procedures was done regards Operative time, wound size, Intraoperative blood loss, Intraoperative complications (nerve root injury or dural tear), postoperative hospital stay, Postoperative complication as wound infection and CSF leakage and the results of VAS and modified MacNab's criteria.

\subsection{Statistics}

The data analyzed using SPSS version 20.0. The qualitative data described by number and percentage, while quantitative data described by mean, range, standard deviation and $95 \%$ confidence interval. 
Pearson Chi square test, fisher exact test and two samples T-test were used to compare different variables. $\mathrm{P}$ value less than 0.05 was considered statistically significant.

\section{Results}

The data of this prospective randomized controlled trial were summerized in Table 1 . This study included 30 patients (18 males and 12 females). The mean age of the two groups of patients was about 35 years. All patients presented with low back pain and unilateral sciatica. 20 patients $(66.66 \%)$ had L4-5 disc, while 10 patients (33.33\%) had L5-S1 disc prolapse. Half of the patients (50\%) were operated on right side and the remainder on the left side.

The mean operative time of COD group was 92 minutes and ranged from 60 to 120 minutes, it was less than PELD group with statistically significant difference $(P=0.014)$ as shown in Table 1 , the longer duration of PELD may be due to the long learning curve of the surgeon.

The length of skin incision is smaller in PELD group (mean $2.63 \mathrm{~cm}$ ) than COD group (mean $4.67 \mathrm{~cm}$ ), this was due to the use of tubular retractor which had a fixed diameter. The mean blood loss was $98.67 \mathrm{cc}$ in PELD group, which was less than COD group with statistically significant difference as shown in Table 2 . It was mostly due to epidural venous bleeding and controlled by gel foam.

Table 1. Distribution of the studied groups according to different parameters.

\begin{tabular}{|c|c|c|}
\hline Parameters & PELD group & COD group \\
\hline Sex (male/female) & $10 / 5$ & $8 / 7$ \\
\hline Age (mean, range) in years & $35.9[26-43]$ & $35.3[21-50]$ \\
\hline \multicolumn{3}{|l|}{ Preoperative clinical presentation (No., \%): } \\
\hline - Back pain & $15(100 \%)$ & $15(100 \%)$ \\
\hline - $\quad$ Sciatica & $15(100 \%)$ & $15(100 \%)$ \\
\hline - $\quad$ Motor deficit & $0(0 \%)$ & $0(0 \%)$ \\
\hline - Sphinecteric disturbance & $0(0 \%)$ & $0(0 \%)$ \\
\hline \multicolumn{3}{|l|}{ Level of disc (No., \%): } \\
\hline - $\quad$ L4-5 & $9(60 \%)$ & $11(73.33 \%)$ \\
\hline - $\quad$ L5-S1 & $6(40 \%)$ & $4(26.66 \%)$ \\
\hline \multicolumn{3}{|l|}{ Side of surgery (No., \%): } \\
\hline - Rt side & $10(66.66 \%)$ & $5(33.33 \%)$ \\
\hline - Lt side & $5(33.33 \%)$ & $10(66.66 \%)$ \\
\hline Duration of surgery in minutes (mean, range) & $124[80-210]$ & $92[60-120]$ \\
\hline Blood loss in cc (mean, range) & $98.67[50-200]$ & $133.33[90-330]$ \\
\hline \multicolumn{3}{|l|}{ Intraoperative complication (No., \%): } \\
\hline - Dural tear & $3(20 \%)$ & $2(12.33 \%)$ \\
\hline - $\quad$ Nerve injury & $0(0 \%)$ & $0(0 \%)$ \\
\hline \multicolumn{3}{|l|}{$\begin{array}{l}\text { Postoperative compilation (No., \%): } \\
\text { - } \quad \text { C. S. F leak } \\
\text { - } \quad \text { Wound infection }\end{array}$} \\
\hline $\begin{array}{ll}\text { - } & \text { Discitis } \\
\text { - } & \text { Pseudomeningocele }\end{array}$ & $0(0 \%)$ & $0(0 \%)$ \\
\hline
\end{tabular}


Table 2. Comparison between different parameters in PELF and COD groups.

\begin{tabular}{|c|c|c|c|c|c|c|c|}
\hline & \multicolumn{3}{|c|}{ PELD } & \multicolumn{3}{|c|}{ COD } & \multirow{2}{*}{$P$ value } \\
\hline & Mean & SD & $95 \% \mathrm{CI}$ & Mean & SD & $95 \% \mathrm{CI}$ & \\
\hline Operative time (min.) & 124 & 35.62 & {$[7.03 / 56.97]$} & 92 & 30.98 & [7.01/56.99] & 0.014 \\
\hline Wound length $(\mathrm{cm})$ & 2.63 & 0.091 & {$[-2.544 /-1.522]$} & 4.67 & 0.232 & {$[-2.557 /-1.509]$} & 0.0001 \\
\hline $\begin{array}{l}\text { Intraoperative blood } \\
\text { loss }(\mathrm{cc})\end{array}$ & 98.67 & 51.67 & {$[-72.25 / 2.92]$} & 133.33 & 48.79 & {$[-72.26 / 2.93]$} & 0.01 \\
\hline Hospital stay (hours) & 33.6 & 12.17 & {$[-17.91 /-1.29]$} & 43.2 & 9.94 & {$[-17.93 /-1.28]$} & 0.025 \\
\hline
\end{tabular}

Intra-operative complications included three patients of dural tears $(20 \%)$ in PELD group; all were repaired using muscle graft and fibrin glue. None of them developed CSF leak or pseudomeningocele. While two patients (12.3\%) had dural tear in COD group and were directly repair by suturing. Also, none of them developed postoperative leak or pseudomeningocele.

No patient had postoperative wound infections or discitis in the follow up period. Most of the patients PELD group discharged from the hospital earlier than COD group as shown in Table 2 .

The mean preoperative VAS of LBP and radicular pain was 6.13 and 6.73 respectively in endoscopic group, while in open group 6.73 and 6.8 respectively. Marked improvement of postoperative VAS was detected with mean (1.6 - 1.9) range for the two groups.

Although, there was postoperative improvement of the VAS and MacNab's criteria in the two groups, there was no statistically significant difference between the preoperative and postoperative VAS of low back pain and radicular pain for the two groups in the follow up period as shown in Table 3.

\section{Discussion}

Different techniques of lumbar discectomy have a success rate of $74 \%$ to $98 \%$ [4]. They focused on nerve root decompression with preservation of the bony and ligamentous structures of the spine.

In our study the mean age was 35.3 years and 35.9 years for open and endoscopic groups respectively. This agreed with Huang et al., 2005 and Schick et al., 2002, where the mean age was 39.7 and 39.5 years respectively [5] [6]. It's noticed that the age range in many similar studies was around 40 years old. This may be explained by the increasing disc degeneration through the activity years from 20 to 40, which are the years of most muscular activity, till the disc prolapses and patient becomes in need for surgery.

Male predominance were found in our study, which agreed with Hermantin et al., [7] who on their study published in 1999 had 39 male (65\%) and 21 female (35\%), which is the same sex distribution percentage of our study. We think that males are more vulnerable to lumbar disc prolapse than females due to more muscular jobs and heavy weight lifting.

Our study showed mean value of operative time for open group was less than 
Table 3. Comparison between pre and postoperative VAS in the 2 groups after 6 months.

\begin{tabular}{|c|c|c|c|c|c|c|c|c|}
\hline & & \multicolumn{3}{|c|}{ PELD } & \multicolumn{3}{|c|}{$\mathrm{COD}$} & \multirow{2}{*}{$P$ value } \\
\hline & & Mean & SD & $95 \%$ CI & Mean & SD & $95 \%$ CI & \\
\hline \multirow{2}{*}{$\begin{array}{l}\text { LBP } \\
\text { VAS }\end{array}$} & Preop. & 6.13 & 0.916 & {$[-1.27 / 0.072]$} & 6.73 & 0.884 & {$[-1.27 / 0.073]$} & \multirow[b]{2}{*}{0.078} \\
\hline & Postop. & 1.6 & 0.632 & {$[-0.728 / 0.328]$} & 1.8 & 0.775 & {$[-0.729 / 0.329]$} & \\
\hline \multirow{2}{*}{$\begin{array}{l}\text { Radicular } \\
\text { pain VAS }\end{array}$} & Preop. & 6.73 & 0.704 & {$[-0.655 / 0.522]$} & 6.80 & 0.862 & {$[-0.656 / 0.523]$} & \multirow{2}{*}{0.445} \\
\hline & Postop. & 1.7 & 0.594 & {$[-0.712 / 0.324]$} & 1.9 & 0.743 & {$[-0.776 / 0.356]$} & \\
\hline
\end{tabular}

endoscopic group with statistically significant difference $(P=0.014)$. As for other series, Dasenbrock et al., 2012 gave operative time 49 minutes for endoscopic and 44 minutes for open discectomy [8]. However, Huang et al., 2005 gave 109 minutes for endoscopic, 72.1 minutes for open discectomy [5]. Significant difference in our study between the two groups can be explained by the long learning curve in endoscopic discectomy, especially in the early cases.

The wound length mean value in our endoscopic group was significantly smaller than conventional open wound. This difference is due to fixed endoscopic tubular retractor diameter used $2.5 \mathrm{~cm}$, and the need in conventional open technique to cut a little bit wider wound for illumination. Also Huang et al., 2005 had nearly similar results [5]. Righesso et al. [9] series had slightly less skin incision length with mean $2.1 \mathrm{~cm}$; range from $1.9-2.3 \mathrm{~cm}$.

Endoscopic intraoperative bleeding amount in our study is significantly less than bleeding of conventional surgery; this can be explained by smaller wound of endoscopic group and good haemostasis. This agreed with Nakagawea et al., 2003 [10] and Huang et al., 2005 [5] where the mean intraoperative bleeding for endoscopic group and open group was about $92.9 \mathrm{cc}$ and $87.5 \mathrm{cc}$ respectively.

Regarding intraoperative complications in our study, dural tears occurred in 3 patients (20\%) in PELD group, and in 2 patients (12.3\%) in COD group. We had no patient $(0 \%)$ of nerve root injury.

All patients with dural tear were repaired intraoperatively by muscle graft and glue or direct suturing. No patient develope postoperative C. S. F leak or pseudomeningocele.

Kullarni et al. [11] had 11 patients (5\%) with dural tears, while Perez-Cruet et al. [12] had 8 patients (5\%) of dural tears and one patient (0.7\%) developed delayed pseudomeningocele, and there were no root injuries. In Teli et al. [13] series, 3\% had nerve root injuries and $8.7 \%$ patients had dural tear, while in Oertel et al. [14] these were no dural tears or nerve root injures. The discrepancy of our results to other series was due to the small sample size.

The length of hospital stay was significantly less for endoscopic group with mean value 33.6 hours due to less immediate postoperative wound pain in endoscopic group. This nearly agreed with Righesso et al. [9] and Katayama et al. [15] who reported 24 hours and 36 hours hospital stay respectively. shorter hospital stay were reported by Choi et al. [16] and Perez-Cruet et al. [12] who re- 
potted 12 hours and 7.7 hours respectively. In Huang et al., 2005 [5], it was 85.7 hours for endoscopic discectomy and 142 hours for open discectomy. It's a longer postoperative hospital stay buy may be those authors prefered to lengthen the immediate postoperative observation period of their patients for study purpose, but we usually discharge the patient as soon as he gets stable with no complications that need more hospitalization. Regarding postoperative complications, in our study there were no (0\%) would infection, discitis, CSF leak o pseudomeningocele. Also, Choi et al. [16] reported no patients of wound infection, while Katayama et al. [15] reported $1 \%$ of wound infection, and Teli et al. [13] reported $1.4 \%$ of postoperative discitis.

In our series, there was a statistically significant difference between the preoperative VAS of LBP and radicular pain (mean 6.13 and 6.73 respectively) and the postoperative VAS (mean 1.6 and 1.7 respectively); with mean follow up period 9.3 months for PELD group ( $\mathrm{P}=0.001)$. Arts et al. [17] had LBP VAS often 1 year for endoscopic surgery 2.25 and for open surgery 2.1 points. In Choi et al. [16] the mean preoperative VAS was 7.89, and postoperatively often 18 months was 1.58. Also, in Righesso et al. [9] the mean preoperative VAS was 7.9 and at the last follow up often 24 months; the mean postoperative VAS was 1.

But, regarding the improvement of postoperative VAS and MacNab's criteria; there was no statistically significant difference between the endoscopic and open group as shown in Table 3. This agreed with Li et al. [18] and Dasenbrok et al. [5].

\section{Conclusion and Recommendation}

Both techniques give good results for patients; each technique has some advantages over the other.

Endoscopic discectomy advantages include smaller wound, results in less intraoperative bleeding, and shortened hospital stay due to less wound pain. On the other hand, conventional open discectomy gives wider wound with good visualization and better range of movement for instruments that makes it easier to detect and deal with any events.

It's well known that endoscopic discectomy takes more time from surgeons to gain experience as it has longer learning curve.

Thus, care must be taken to wisely make the right decision for every patient and choose the best surgical technique, which must be most appropriate for the patient and well experienced by the surgeon.

Limitation of our study was the small sample size and short duration, so we recommend to increase the sample size and longer time to evaluate the long term results of the procedures.

\section{Conflicts of Interest}

The authors declare no conflicts of interest regarding the publication of this paper. 


\section{References}

[1] Saal, E. and Saal, M. (1992) Intradiscal Electrothermy, Managining Lumbar Disc Herniation. Spine, 1, 1239.

[2] Casper, W., Campbell, B., Barbier, D.D., Kretschmmer, R. and Gotfried, Y. (1991) The Caspar Micro-Surgical Discectomy and Comparison with a Conventional Standard Lumbar Disc Procedure. Neurosurgery, 28, 78-87. https://doi.org/10.1227/00006123-199101000-00013

[3] Lee, D.Y., Shim, C.S., Ahn, Y., Choi, Y.G., Kim, H.J. and Lee, S.H. (2009) Comparison of Percutaneous Endoscopic Lumbar Discectomy and Open Lumbar Micro-Discectomy for Recurrent Disc Herniation. Journal of Korean Neurosurgical Society, 46, 515-521. https://doi.org/10.3340/jkns.2009.46.6.515

[4] Cenic, A. and Kachur, E. (2009) Lumbar Discectomy: A National Survey of Neurosurgeons and Literature Review. Canadian Journal of Neurological Sciences, 36, 196-200. https://doi.org/10.1017/S0317167100006557

[5] Huang, T.J., Hsu, R.W., Li, Y.Y. and Cheng, C.C (2005) Less Systemic Cytokine Response in Patients Following Micro-Endoscopic versus Open Lumbar Discectomy. Journal of Orthopaedic Research, 23, 406-411. https://doi.org/10.1016/j.orthres.2004.08.010

[6] Schick, U., Dohnert, J., Richter, A., Konig, A. and Vitzthum, H.E. (2002) Micro-Endoscopic Lumbar Discectomy versus Open Surgery: An Intraoperative EMG Study. European Spine Journal, 11, 20-26. https://doi.org/10.1007/s005860100315

[7] Hermantin, F.U., Peters, T., Quartararo, L. and Kambin, P. (1999) A Prospective, Randomized Study Comparing the Results of Open Discectomy with Those of Video-Assisted Arthroscopic Microdiscectomy. JBJS, 81, 958-965.

https://doi.org/10.2106/00004623-199907000-00008

[8] Dasenbrock, H.H., Juraschek, S.P., Schultz, I.R., Witham, T.F., Sciubba, D.M., Wolinsky, J.P., Gokaslan, Z.L. and Bydon, A. (2012) The Efficacy of Minimally Invasive Discectomy Compared with Open Discectomy: A Metal-Analysis of Prospective Randomized Controlled Trials. Journal of Neurosurgery, 16, 452-462. https://doi.org/10.3171/2012.1.SPINE11404

[9] Righesso, O., Falavigna, A. and Avanzi, O. (2007) Comparison of Open Discectomy with Micro-Endoscopic Discectomy in Lumbar Disc Herniations: Results of a Randomized Controlled Trial. Neurosurgery, 61, 545-549. https://doi.org/10.1227/01.NEU.0000290901.00320.F5

[10] Nakagawa, H., Kamimura, M., Uchiyama, S., Takahara, K., Itsubo, T. and Miyasaka, T. (2003) Micro-Endoscopic Discectomy (MED) for Lumbar Disc Prolapse. Journal of Clinical Neuroscience, 10, 231-235. https://doi.org/10.1016/S0967-5868(02)00337-5

[11] Kulkarni, A.G., Bassi, A. and Dhruv, A. (2014) Micro-Endoscopic Lumbar Discectomy: Technique and Results of 188 Cases. Indian Journal of Orthopedics, 48 , 81-87. https://doi.org/10.4103/0019-5413.125511

[12] Perez-Cruet, M.J., Foley, K.T., Isaacs, R.E., Rice-Wyllie, L., Wellington, R., Smith, M.M., et al. (2002) Micro-Endoscopic Lumbar Discectomy: Technical Note. Neurosurgery, 51, 129-136. https://doi.org/10.1097/00006123-200211002-00018

[13] Teli, M., Lovi, A., Brayda-Bruno, M., Zagra, A., Corriero, A., Giudici, F., et al. (2010) Higher Risk of Dural Tears and Recurrent Herniation with Lumbar Micro-Endoscopic Discectomy. European Spine Journal, 19, 443-450.

https://doi.org/10.1007/s00586-010-1290-4 
[14] Oertel, J.M.K., Mondorf, Y. and Gaab, M.R. (2009) A New Endoscopic Spine System: The First Results with "Easy GO". Acta Neurochirurgica, 151, 1027-1033. https://doi.org/10.1007/s00701-009-0454-7

[15] Katayama, Y., Matsuyama, Y., Yoshihara, H., Sakai, Y., Nakamura, H., Nakashima, S., et al. (2006) Comparison of Surgical Outcomes between Macro Discectomy and Micro Discectomy for Lumbar Disc Herniation: A Prospective Randomised Study with Surgery Performed by the Same Spinal Surgeon. Journal of Spinal Disorders \& Techniques, 19, 344-347. https://doi.org/10.1097/01.bsd.0000211201.93125.1c

[16] Choi, G., Lee, S.-H., Raiturker, P.P., Lee, S. and Chae, Y.S. (2006) Percutaneous Endoscopic Interlaminar Discectomy for Intracanalicular Disc Herniations at L5-S1 Using a Rigid Working Channel Endoscope. Neurosurgery, 58, 59-68.

[17] Atrs, M.P., Brand, R., Van Den Akker, M.E., Koes, B.W., Bartels, R.H., Tan, W.F. and Peul, W.C. (2001) Tubular Diskectomy vs. Conventional Microdiskectomy for the Treatment of Lumbar Disk Herniation: 2-Year Results of a Double-Blind Randomized Controlled Trial. Neurosurgery, 69, 135-144.

[18] Li, S.H., Li, H.Z. and Zhao, J.R. (2008) Clinical Comparison between Micro-Endoscopic Discectomy (MED) and Open Discectomy for Treatment of Lumbar Disc Herniation. China Journal of Orthopaedics and Traumatology, 21, 349-351. 